\title{
Modificación técnica de la desinserción ureteral endoscópica en la nefroureterectomía
}

\author{
Aguirre Benites F, Blanco Carballo O, Pamplona Casamayor M, Díaz González R, Leiva Galvis O. \\ Servicio de Urología. Hospital Universitario 12 de Octubre. Madrid. \\ Actas Urol Esp. 2007;31(7):785-787
}

\section{RESUMEN}

\section{MODIFICACIÓN TÉCNICA DE LA DESINSERCIÓN URETERAL ENDOSCÓPICA} EN LA NEFROURETERECTOMÍA

Se presenta una variante técnica de la desinserción endoscópica ureteral, con la que se intenta evitar el contacto de la orina con el lecho quirúrgico, condición preceptiva para evitar la posible diseminación tumoral local, al realizar la nefroureterectomía radical en tumores de urotelio superior.

Palabras clave: Nefrourterectomía.Desinserción ureteral endoscópica. Modificación técnica.

\section{ABSTRACT \\ ENDOSCOPIC MODIFIED TECHNIQUE OF URETERAL RESECTION DURING NEPHROURETERECTOMY}

We show a technical modification of the ureteral endoscopic resection with which we try to avoid comunication between urine and surgical bed in order to prevent tumor local spread of upper urotelial tumor.

Keywords: Nephrouerterectomy. Endoscopic ureteral resection. Technique.

$\mathrm{L}$ a nefroureterectomía total, ya sea mediante cirugía abierta con abordaje lumbar e ileoinguinal, o por laparoscopia, es la opción terapéutica de elección en los tumores uroteliales de tracto urinario superior, excepto en aquellos casos en los que esté indicado un tratamiento consevador (pacientes monorrenos, tumores bilaterales, insuficiencia renal y tumores de bajo grado y estadio).

La intervención exige desde el punto de vista oncológico la exéresis, de la totalidad del uréter y de un rodete vesical perimeático ipsilateral siendo preceptivo evitar el contacto de la orina de la vía excretora con el lecho quirúrgico.

\section{CASO CLINICO}

Varón de 67 años con antecedentes de hipertensión y hernia de hiato en tratamiento médico que acude a la consulta de urología por hematuria monosintomática y autolimitada en el tiempo.
La exploración física no reveló hallazgos de interés.

En las determinaciones analíticas sanguíneas todos los parámetros se encontraban en los límites de la normalidad.

Se le realiza urografia intravenosa observando un defecto de replección en pelvis renal derecha que posteriormente se confirma con una tomografia computerizada abdominal que muestra una masa en pelvis que amputa cáliz medio.

Las citologias eran negativas.

Se realizó nefroureterectomía derecha. En un primer tiempo se procede a desinserción ureteral endoscópica con la modificación técnica posteriormente descrita para a continuación llevar a cabo la nefroureterectomía a través de una incisión subcostal derecha.

En la descripción macroscópica de la pieza se aprecia una lesión vegetante, exofítica y papilar que ocupa el cáliz medio y parte de pelvis renal sin infiltrar parénquima. 
Microscópicamente el tumor es informado como carcinoma urotelial de alto grado que no invade la lámina propia (Ta G3).

Tras cuatro años de seguimiento el paciente se encuentra vivo y libre de enfermedad.

\section{TECNICA GUIRURGICA}

La resección del meato ureteral hasta la grasa con desinserción del uréter endoscópicamente, constituye un método rápido en el tratamiento del uréter distal, pero la posibilidad de paso de orina de la vía excretora al lecho quirúrgico con el riesgo de diseminación tumoral, ya descrita ${ }^{1,2}$ la ha puesto en entredicho.

Presentamos una modificación técnica del método de desinserción ureteral endoscópico que suprime el riesgo de diseminación tumoral en el lecho operatorio a la vez que facilita el acto quirúrgico.

Con el paciente en posición de litotomía se realiza una cistoscopia para descartar la presencia de tumor vesical concomitante.

Una vez descartado, se localiza el meato ureteral correspondiente a la vía afectada por el tumor e introduciendo una guía teflonada hasta la pelvis renal, se retira el cistoscopio y paralelo a la guía teflonada se introduce un resector de calibre $26 \mathrm{CH}$ que se monta con un asa de Collins.

A continuación y utilizando la guía teflonada como tutor se pasa al uréter una sonda balón comprobando bajo visión que el balón queda situado en la porción intramural del uréter de tal forma que al rellenarlo, su extremo distal aparece unos milimetros por fuera del meato ureteral (Fig. 1).

Se procede entonces, garantizada la estanqueidad de la vía urinaria, a la desinserción ureteral mediante incisión con el asa de Collins del área perimeática. Profundizamos la misma, liberando la totalidad del uréter intramural hasta la grasa. Esta maniobra es fácil de realizar, y evita el abordaje transvesical ya que el balón hinchado dentro del uréter intramural lo delimita perfectamente y permite al traccionar de la sonda balón su rápida disección.

Terminamos colocando una sonda de Foley $n^{\circ}$ 18 en vejiga que recogerá la orina contralateral (Figs. 2, 3, 4 y 5).

A continuación realizamos la nefrectomía mediante cirugía abierta o laparoscópica, colocando un clip en el uréter distal inmediatamente por encima del extremo proximal del balón, deshinchamos el balón y extraemos la pieza quirúrgica.

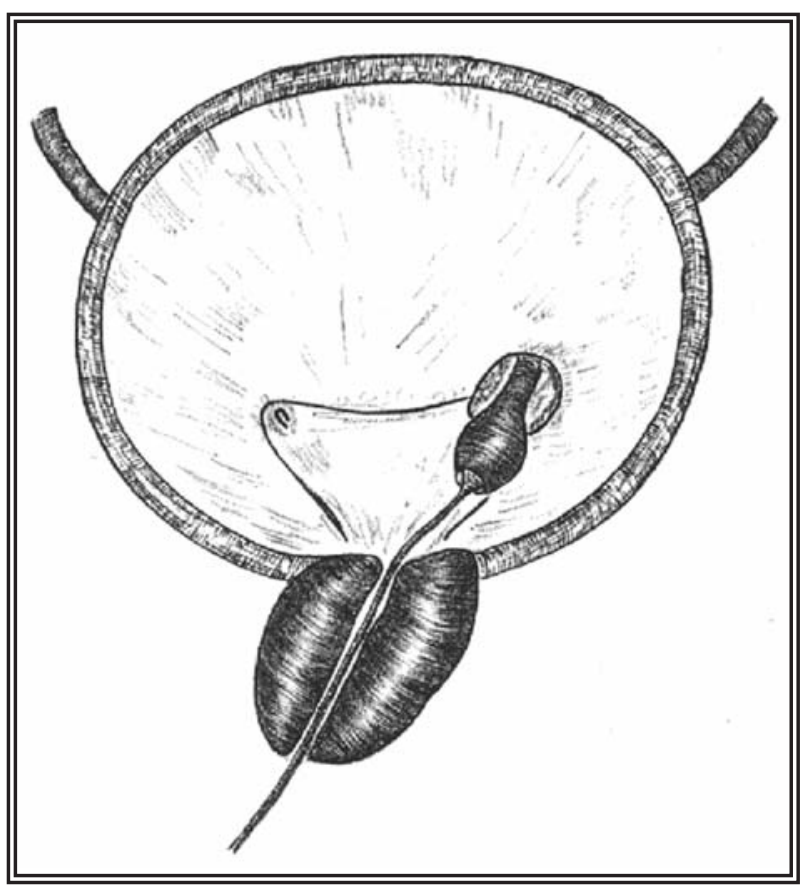

FIGURA 1. Esquema de la situación inicial.resector $N^{\circ} 26$ montado con asa de Collins y sonda balón introducido en el uréter.

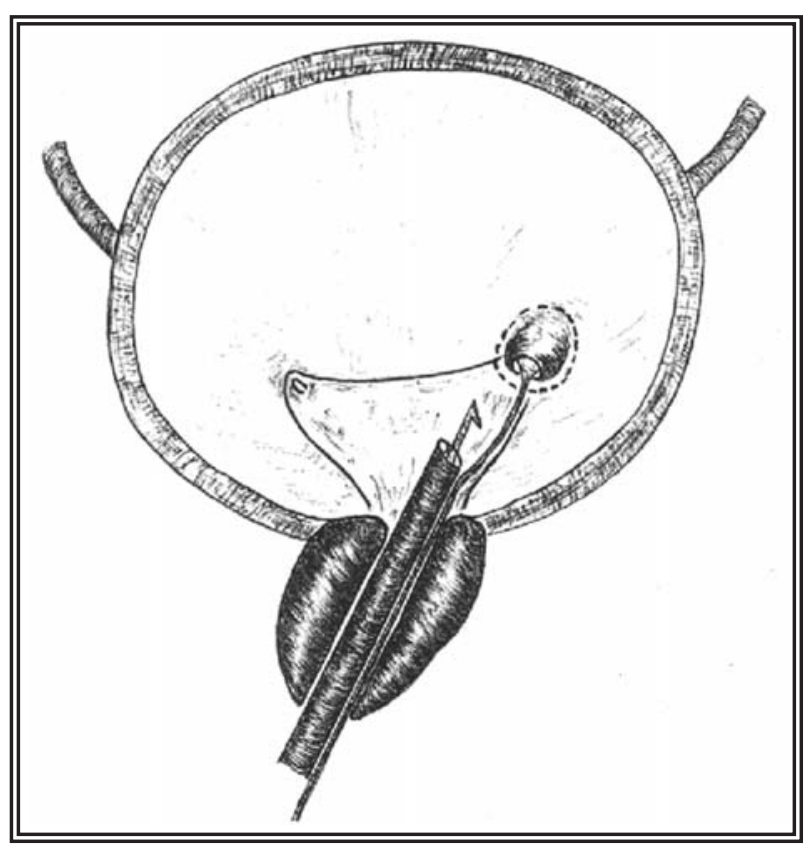

FIGURA 2.Ureter desinsectado manteniendo la sonda balón evitando fugas urinarias.

\section{DISCUSION}

El tratamiento estándar en el tumor urotelial de tracto urinario superior es la nefroureterectomía radical con exéresis de manguito vesical ya que se ha encontrado una incidencia aproximada 


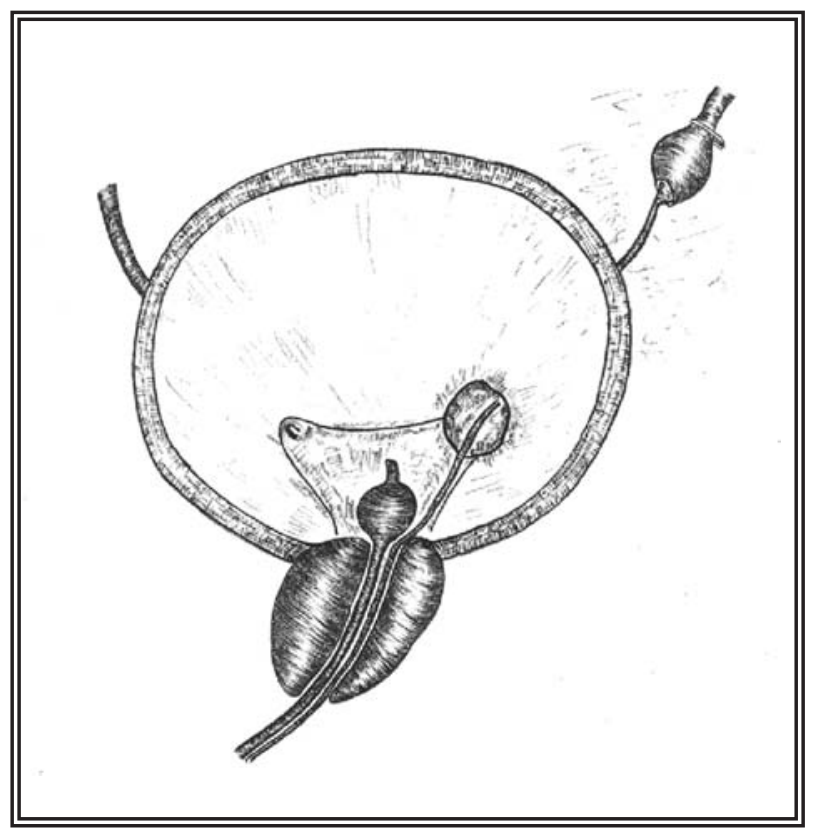

FIGURA 3. Esquema de la situación final. Uréter desinsectado y clampado en situación extravesical, manteniendo sonda Foley $n^{\circ} 18$ como drenaje vesical.

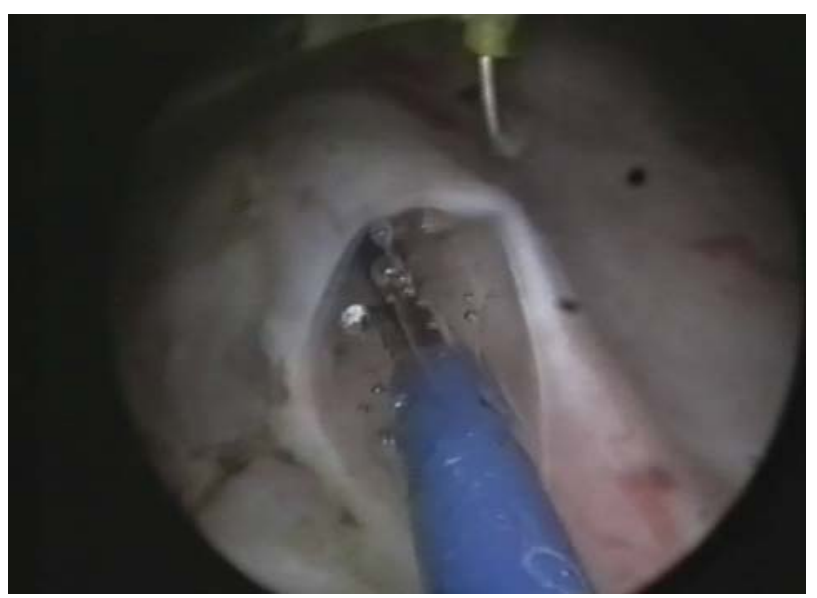

FIGURA 4 Sonda balón introducida en el uréter y asa de Collins relizando desinserción.

entre el $50-70 \%$ de recurrencia unilateral o próximo al orificio ureteral cuando se realiza tratamiento conservador.

La técnica clásica se define por una incisión de lumbotomía acompañada de una incisión inguinal ipsilateral siendo factible la realización de laparotomía media o lumbotomía prolongada.

En la década de los 80 se realiza por primera vez la técnica de desinserción ureteral como técnica válida y reproducible para el tratamiento de tumores de tracto urinario superior localizados en pelvis y cálices.

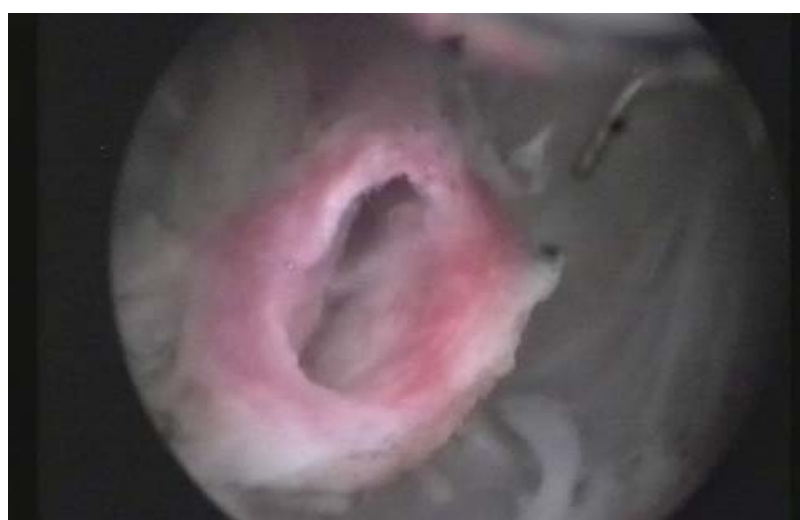

FIGURA 5. Meato desinsertado.

Desde entonces se han publicado múltiples series destacando la necesidad de evitar la realización de esta técnica en tumores de localización en uréter distal o meato ureteral para evitar la posible diseminación tumoral mientras se realiza la nefrectomía.

Se ha descrito en casos aislados la recidiva de tumor urotelial en el lugar de la desinserción.

Los beneficios de esta técnica son la disminución del tiempo quirúrgico, menor tasa de infecciones de herida quirúrgica así como menor dolor postoperatorio.

Hemos tratado 9 pacientes con esta modificación técnica de desinserción endoscópica del uréter previa a la nefroureterectomía, que presentaban tumores pielocaliciales, no habiendo presentando en ningún caso recidiva tumoral en el lecho quirúrgico extravesical ni en el área trigonal de la desinserción, después de un seguimiento de 5 años.

Creemos, basados en nuestra experiencia, que la aplicación de esta modificación técnica de la desinserción ureteral endoscópica en la nefroureterectomía total por tumor urotelial pielocalicial, ya sea mediante cirugía abierta o laparosocopia, facilita la realización de la misma, evita la posible diseminación tumoral en el lecho quirúrgico y permite la aplicación incluso en los tumores de localización ureteral situados por encima del cruce de los vasos iliacos.

\section{REFERENCIAS}

1. Arango O, Bielsa O, Carles J, Gelabert-Mas A.Massive tumor implantation in the endoscopic resected area in modified nephroureterectomy. J.Urology;1997 157(5):1839.

2. Hetherington JW, Ewing R, Philp NH. Modified nephroureterectomy: A risk of tumor implantation. Brit J Urol. 1986;58(4): 368-370.

Correspondencia autor: Dr. F. Aguirre Benites

Servicio de Urología. Hospital Universitario 12 de Octubre.

Avda. de Córdoba, s/n - 28041 Madrid. Tel.: 913908121

Información artículo: Nota clíinica

Trabajo recibido: enero 2006

Trabajo aceptado: septiembre 2006 